the same feeling when reading this book, which is rather odd, since it purports to (deep breath here) "provide information to those multidisciplinary professionals who work in mental health and have little awareness and/or appreciation of the prevalence of various forms of communication breakdown in people with mental illness and therefore the place that the speech and language therapist (SLT) might have in helping to alleviate these problems". Only the difficulty is that I still have not reached the "Aha, so that's what it is" stage. Maybe it is me. Maybe not.

I am not saying that the book is not scattered with undoubted gems. I should mention here David Newby's concise chapter on communication and formal thought disorder in schizophrenia and a fascinating contribution by Alice Thackery entitled "What can we learn from the deaf patient?" But the problem may be that the editors had two quite different aims: selling the idea of the SLT as a member of the multi-disciplinary team to mental health professionals, and selling the ideas of working in mental health to SLTs and their students. Unfortunately, neither of these laudatory aims is satisfactorily fulfilled.

Psychiatrists working in rehabilitation, who probably have the most to gain from an attached SLT, would have to search quite hard here to discover exactly how an SLT would provide 'added value' to their team. SLTs and their students who are new to mental health will not learn a great deal about modern approaches to assessment and treatment from the early chapters of this book, which are curiously quaint in places. There is, for example, no mention of selective serotonin reuptake inhibitors in the chapter on the treatment of depression. Personal construct psychology gets a whole chapter, while cognitive-behavioural therapy is afforded only a passing mention. The declamatory style of writing is best conveyed by the statement "few personality disordered people excel academically". Those of us who work in universities might disagree here.

I must note, however, that people who specialise in communication can fail to convey ideas clearly - not just those with formal thought disorder.

Linda Gask Reader and Honorary Consultant Psychiatrist, University of Manchester Department of Community Psychiatry, Guild Academic Centre, Royal Preston Hospital, Sharoe Green Lane, Preston PR2 9HT, UK

\section{Comprehensive Care of Schizophrenia: A Textbook of Clinical Management}

Edited by Jeffrey A. Lieberman \& Robin M. Murray. London: Martin Dunitz. 2000. 256 pp. $€ 46.95$ (hb). ISBN I 853178934

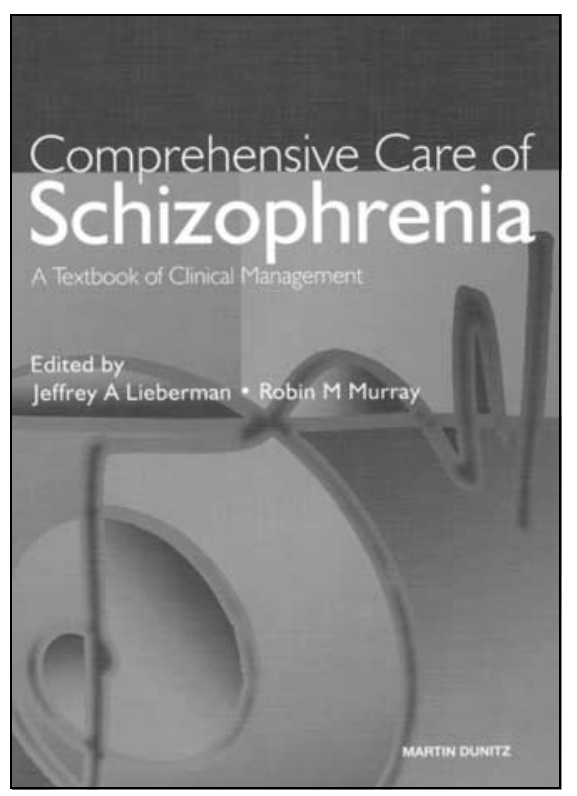

If you have only $£ 50$ left in your library budget, buy this book. It is the best and most comprehensive textbook for the clinical management of schizophrenia that I have seen in a very long time. The attractive layout, exceptionally clear language and beautiful printing makes the book so easy and enjoyable to read that it could even be recommended for bedside reading. The deceptively user-friendly style disguises a wealth of information on evidence-based clinical practice, practical advice and thoughtful opinion where sufficient evidence is lacking. The text belies the adage that incomprehensibility is a sign of authority, since the authors convey complex aspects of diagnosing and managing schizophrenia in an easily accessible format suitable for a wide spectrum of mental health professionals.

The book covers all aspects of managing schizophrenia, discussing not only different stages of the illness but also special issues such as violence, schizophrenia in women and different systems of care in the developing and the developed world. There are very useful checklists, guidelines and diagrams, for both planning treatment and understanding biological aspects such as neuroreceptor binding affinities of antipsychotic drugs. One chapter covers the importance of the clinicianpatient and clinician-carer relationships, not only in the illness in general but also during specific phases such as the prodrome. Clinicians in the UK continue to delay making and communicating the diagnosis of schizophrenia to patients and families, which can often cause unnecessary anguish and delay in treatment. This chapter advises clinicians how to handle and communicate uncertainty to patients and families, not necessarily by minimising the gravity of what might unfold. There are excellent chapters on childhood- and adolescent-onset schizophrenia, medical management of patients with schizophrenia, treating chronic schizophrenia and violence in the acute and chronic phases. The penultimate chapter is a series of firstperson accounts. The chapters are all of a high standard and some, such as that on cognitive-behavioural therapy, are particularly good.

I can find no shortcomings in this book. Community mental health teams would be well advised to invest in it since it will be useful for clinicians from all disciplines and, given its up-to-date reference list, is unlikely to be superseded in the near future.

Swaran P. Singh Consultant Psychiatrist and Senior Lecturer, Community Psychiatry, St George's Hospital Medical School, Jenner Wing, Cranmer Terrace, London SWI7 ORE, UK

\section{Ethics Primer of the American Psychiatric Association}

American Psychiatric Association. Washington, DC: APA. 200I. 102 pp (pb). ISBN 0890423172

This is a most curious publication. The word primer is derived from the Latin primus (first) and usually refers to a textbook that provides the first principles of the subject. Ethics Primer certainly does not do this. The slim volume comprises 11 chapters and an appendix containing the 2001 edition of the Principles of Medical Ethics with Annotations Especially Applicable to Psychiatry (which has been published in many versions by the American Psychiatric Association (APA) since 1973).

Most of the authors are associated with the APA's Ethics Committee and deal with topics within their expertise. When tackling 


\section{Ethics Primer}

American Psychiatric Association

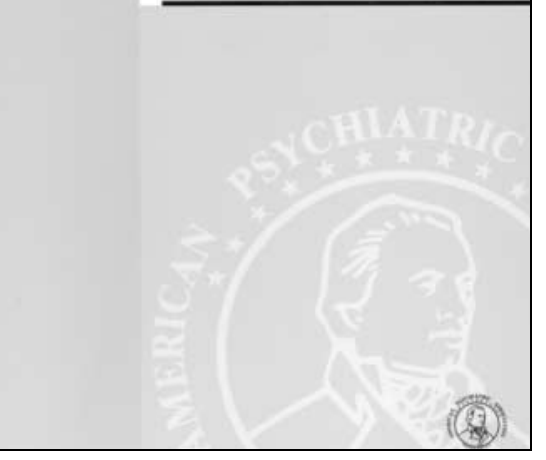

their contributions, I became quite baffled as to what the Committee had in mind when planning and preparing the book. The selection of subjects is not only arbitrary but fails to address many contemporary ethical matters that warrant our attention. Indeed, some of the material covered seems of much lesser relevance. For example, why is there a chapter devoted to gifts, particularly when these are partially dealt with in the chapter on boundary violations? Presentation of material is another problem: the chapter on consultations and second opinions is particularly nebulous.

Further difficulties arise when one tries to detect a common aim. The author of the chapter on children, adolescence and families offers a personal view about the range of issues that arise in this area, whereas the writer of the chapter on involuntary hospitalisation highlights controversies. In another pair of chapters, the authors apply the APA guidelines specifically in dealing with the ethical quandaries on which they focus. After a careful reread, the nagging question remained as to whether this book is intended to be prescriptive, descriptive, a mixture of both, or something else.

Similar criticisms apply to the bibliographies. Some chapters have none; in others they are scanty or dated. A suggested reading list is offered but by only one auther. Again, I wonder what lay behind the editors' thinking when commissioning the contributions.
Having been a proponent of highlighting psychiatric ethics in the professional life of psychiatrists for over two decades, I am always excited about new contributions. Alas, I cannot respond in this way on this occasion and suggest that should the APA consider a second edition it be done with substantially more preparation and planning.

Sidney Bloch Professor of Psychiatry, University of Melbourne, St Vincent's Hospital, Department of Psychiatry, Fitzroy, Victoria 3065, Australia

\section{Comprehensive Guide to Interpersonal Psychotherapy}

By Myrna M.Weissman, John C. Markowitz \& Gerald L. Klerman. New York: Basic Books. 2000.488 pp. $€ 42.50$ (hb). ISBN 0465095666

Interpersonal therapy (IPT) has established itself as a highly effective psychological treatment for most forms of depression. It is a very researchable and teachable one too. Developed within mainstream US psychiatric practice, it works within a traditional diagnostic model. Its range of applications is continuing to expand. Interpersonal therapists need an ability to formulate, to empathise and to facilitate emotional self-expression. This makes IPT an attractive and useful model for nearly all psychiatrists to learn, irrespective of previous psychotherapeutic experience.

Prior to the publication of this excellent volume, the key text was Klerman et al's (1984) detailed practical guide to IPT technique. Nearly all of it is to be found here, comprising about half the present book. Additional material is of three kinds. First, there is some new material on basic methods, including an extended survey of techniques for use in work with couples. Second, there are detailed practical notes on additional clinical applications, including its use in the elderly and adolescents; dysthymia and bipolar disorders; depression associated with childbirth and HIV; and substance misuse, anxiety and eating disorders. Third, the use of IPT in new formats is discussed, including brief counselling, maintenance treatment, groups and telephone contact. Notes on training resources are also updated.

The notes on IPT technique are always clear and relevant. They are illustrated with case examples, several of which are new.
One questionable revision is the now perfunctory discussion of combined treatment with IPT and antidepressants. This assumes that combined treatment presents no significant problems to either patients or therapists and omits basic advice on how combined treatments should be timed. The implications of research seem insufficiently explored here, while a complex clinical issue is excessively simplified. In general, relevant research is introduced without obscuring the book's eminently practical focus, ensuring a long shelf-life. Production maintains the high standards customary in the USA. This 'comprehensive guide' lives up to its name and is now the book to have on IPT. No psychiatric library can be without it.

Klerman, G. L., Weissman, M. M., Rounsaville, B. J., et al (1984) Interpersonal Psychotherapy of Depression. New York: Basic Books.

Chris Mace Consultant Psychotherapist, South Warwickshire Combined Care NHS Trust,

Psychotherapy Service, Yew Tree House, 87 Radford Road, Leamington Spa CV3I IJQ, UK, and senior lecturer in psychotherapy, University of Warwick

\section{Brain Imaging in Schizophrenia: Insights and Applications}

ByTonmoy Sharma \& Xavier Chitnis. London: ReMedica Publishing. 2000. 74 pp. $£ 15.00$ (pb). ISBN I 901346080

\section{BRAIN IMAGING in SCHIZOPHRENIA}

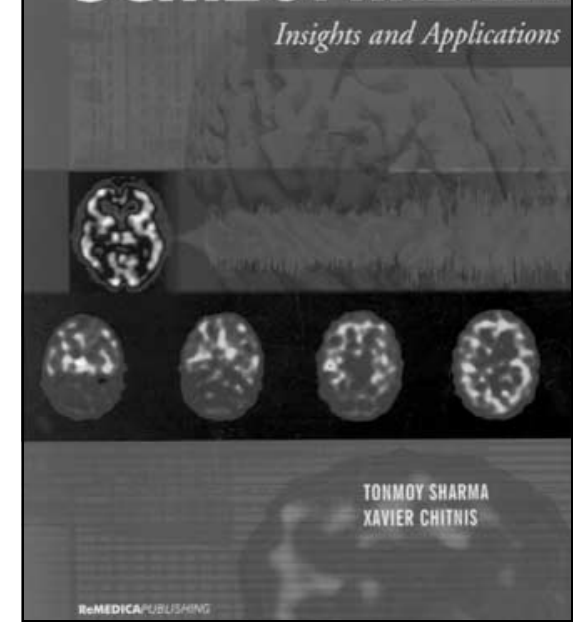

